\title{
The Energy Conservation Design Strategy on Taiyuan South Railway Station Building
}

\author{
Fang Wang ${ }^{1, a^{*}}$ and Li Wang ${ }^{2, b}$ \\ ${ }^{1}$ School of Urban Construction, Wuhan University of Science and Technology, Wuhan 430000, \\ China; \\ ${ }^{2}$ Central-South Architectural Design Institute Co, Ltd, Wuhan 430000, China \\ a6978893@qq.com, b10659648@qq.com \\ *The corresponding author
}

\begin{abstract}
It's always an important guiding principal about how to combine the features of regional climatology and of culture to optimize building that is environmental suitability. It sets Taiyuan south railway station as an example, discussing the influences of natural ventilation, natural lighting and green illumination to it, and the radiant heating systems and intelligent control system are also considered. Besides, as to large space building designing, we using the way of numerical simulation to analysis. The result of this paper can provide significant engineering guide lines to the green building design that is climatic adaptation to some extent.
\end{abstract}

Keywords: The feature of regional climatology; Environmental suitability; Energy conservation design; Numerical simulation; Green building

\section{太原火车站南站建筑节能设计策略$$
\text { 王芳 }{ }^{1} \text {, 王力 }{ }^{2}
$$

(1. 武汉科技大学 城市建设学院, 湖北 武汉 430000;

2. 中南建筑设计院股份有限公司, 湖北 武汉 430000)

摘要: 结合区域气候特征与文化特征, 进行环境适应性的建筑设计是绿色建筑设计的重要指导思想之一。以太原火车站 南站站房设计为例, 从自然通风、自然采光与绿色照明、节能围护结构、地板热辐射采暖以及智能控制几方面展开探讨, 并 采用数值模拟对大空间绿色建筑设计进行分析。作为绿色建筑设计的尝试, 为气候适应性的绿色建筑设计提供了思路与方向。

关键词：地域气候特征；环境适应性；节能设计；数值模拟；绿色建筑

中图分类号：TU248.1 文献标志码：A

引言

面对日益恶化的全球环境局势，低碳化、绿色化逐渐成为各个领域的主流发展方向。而在所有建筑类 型中, 大空间公共建筑因其巨大的空间尺度, 使其在面临建筑与环境、能源等问题上比一般建筑要多得多。 怎样为大空间公共建筑创造良好的自然采光、通风条件及合理的遮阳系统等问题, 是绿色建筑发展的重要 方面之一 ${ }^{[1]}$ 。以太原火车站南站建筑设计为例, 从环境与设计的角度出发, 展开相关的大空间绿色建筑设 计实践。

1 设计思路与目标

太原市由于其地理环境影响, 造成其气候干燥, 降雨偏少, 昼夜温差大的气候特征。太原南站位于太 原市小店区, 设计充分考虑当地气候环境, 采用线侧与高架相结合的布局, 客运用房建筑面积为 $60188 \mathrm{~m}^{2}$; 平面设计分为高架候车层、站台层及地下出站层三层。太原南站从节能减排在建筑技术及构造方面做出了 
许多努力, 以建筑的屋面系统为例, 站房结构单元上部设计通风塔, 通过自动控制电动百叶的设置, 能实 现自然通风及火灾时的排烟; 屋面设置 X 状 PC 板采光天窗, 均匀分布, 尺寸合理以满足自然采光的需求 为准; 主站房屋顶东西向悬挑形成良好的遮阳效果。此外, 建筑大部分外墙为幕墙系统, 由低辐射玻璃, 可控玻璃窗及双层仿清砖石材面板组成，可确保夏季开启、冬季关闭状态，而形成夏季通风、冬季蓄热的 室内环境 ${ }^{[2]}$ 。

\section{2 绿色设计策略}

\section{1 自然采光与绿色照明}

太原南站主站房作为铁路客运站的主要使用空间，人流量集中，空间体量巨大，仅仅利用立面玻璃幕 墙的采光难以满足大进深室内空间的采光需要, 因此, 在设计中结合结构单元体的布局基础上, 借鉴了中 国传统民居中 “亮瓦” 的形式，屋顶设置 X 状的采光天窗 (见图 1)。在每个单元体的屋顶设置了 “X” 形 的半透明高强聚碳酸酯采光天窗, 可将直射阳光过滤为均匀柔和的室内光线, 从而大大的降低了白天室内 的采光能耗。采光天窗部分采用 PC (聚碳酸酯) 板天窗, 在保证其基本屋顶热工基础上, 室外自然光线可 均匀照亮建筑内部, 满足候车大厅站房部分的白天自然采光要求 ${ }^{[3]}$ 。

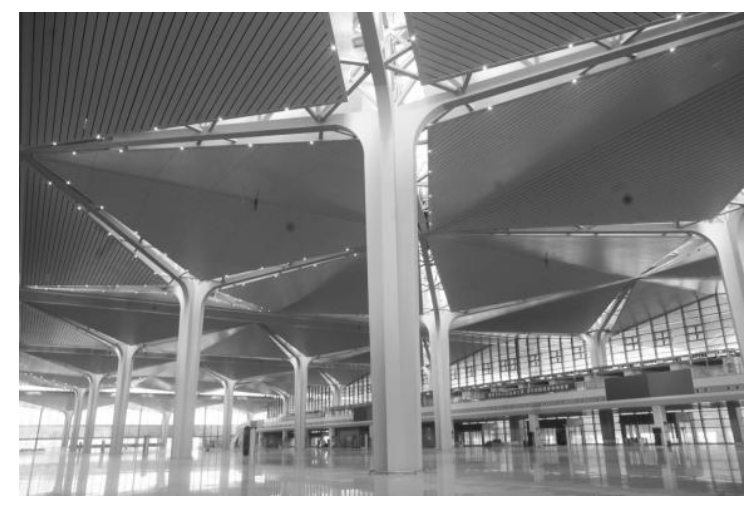

图 1 屋顶 $\mathrm{X}$ 状采光天窗

在人工照明方面，针对候车空间进行自然采光与照明质量的仿真模拟分析，全晴天时室内照度可达到 $6001 x$ 左右。针对候车厅照明设计要求, 在技术设备上采用感光调节措施, 节约照明用电; 并利用混合照 明的形式, 获得良好的视觉舒适性, 充分实现绿色照明与节能设计要求 ${ }^{[4]}$ 。

\section{2 自然通风设计}

在建筑的通风设计中，小空间的自然通风更容易实现，而对于大空间的通风问题实现自然通风难度较 大, 常规的方法是采用空调送风设备来解决。在太原南站的设计中, 将大空间的自然通风技术作为重要的 绿色设计技术之一展开相关的设计与研究。

根据太原地区的气候特征和周围环境状况，考虑在春秋过渡季节和夏季部分适宜时段通过自然通风实 现建筑内空气置换，保证建筑室内的热环境舒适性，从而缩短空调的运行时间，达到建筑节能 ${ }^{[5]}$ 。建筑设 计中, 结合建筑布局与使用功能, 在东西广场入口部分设置可开启的入口门厅, 候车大厅南北向设置可自 动控制的下旋式窗和百叶窗; 同时, 在候车大厅的设计单元上空设置可自动控制的 “风帽”。在适宜季节, 新鲜空气可通过开启的门窗导入, 利用风压和热压, 实现建筑内的空气流动, 实现自然通风 ${ }^{[6]}$ 。 
在建筑的自然通风设计中，采用CFD软件对建筑在环境中的通风效果进行了模拟（见图2）。从模拟的 结果来看, 建筑在整体风环境中的效果较好, 建筑前后形成了明显的风压, 对于建筑内的自然通风有利。 从建筑内部的通风分析模拟来看, 开口的设置对于气流的导入有一定的效果, 风帽位置也有相应空气流动。

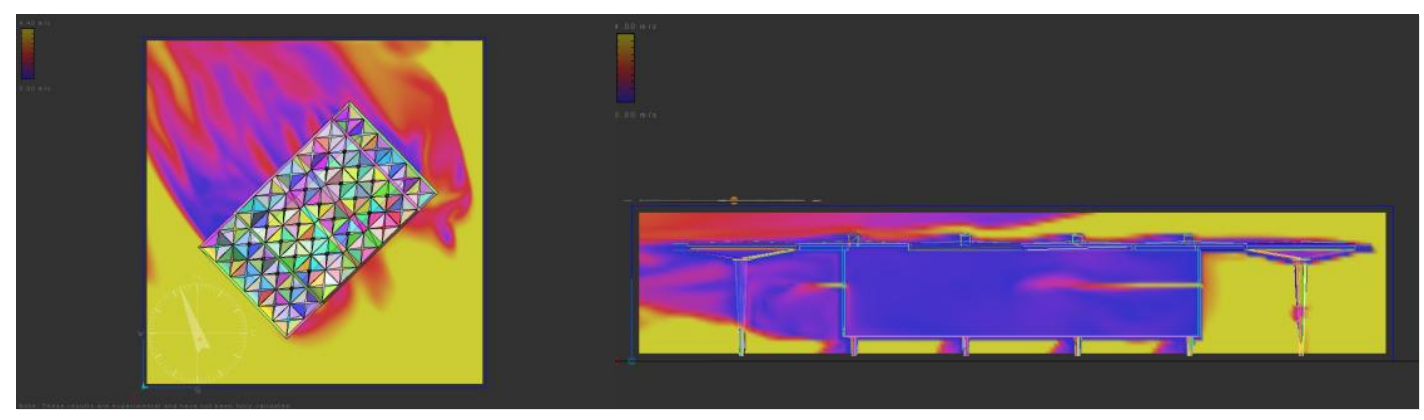

图2 站房自然通风模拟分析

结合相应的通风模拟，在实际建筑中进行了相应的现场实验测试。从部分测试结果来看，东西广场入 口位置风速可达 $1.8 \mathrm{~m} / \mathrm{s}$ 左右, 南北检票口位置最大风速可达 $1.5 \mathrm{~m} / \mathrm{s}$, 南北立面的幕墙开口位置最大风速可 达 $0.3 \mathrm{~m} / \mathrm{s}$ 左右, 而在屋顶风帽口位置风速可达 $0.6 \mathrm{~m} / \mathrm{s}$, 且在建筑的大空间内部出现了明显的温度梯度。初 步的数据表明, 在太原南站的建筑空间内部存在风压通风和热压通风现象, 对于建筑的大空间通风有一定 的帮助作用。

\section{3 节能围护结构体系}

在围护结构节能设计中, 结合气候环境特征与标准要求, 针对围护结构体系进行相应的节能设计。

\subsection{1 屋面系统}

新太原南站屋面主要采用成型铝合金屋面系统，构造层中包含保温矿面层，可起保温作用并有一定隔 音效果; 主站房屋顶天窗采用 $\mathrm{PC}$ 高强度耐候板, 屋顶单元体为平均厚度 $3 \mathrm{~m}$ 的双层结构, 具有极佳的保温隔 热性能, 非常适用于太原地区冬季寒冷的环境特点。此外, 在屋面布置预留安装太阳能板的可能性, 远期 可通过系统将太阳能转换成电能, 为站房提供照明用电 ${ }^{[7]}$ 。

\subsection{2 围护结构}

太原南站外围护体系采用新颖的双层中空玻璃石材组合幕墙, 不仅在造型上继承了山西民居清砖砌筑 的神韵, 同时通过独特的双层玻璃石材组合幕墙构造方式, 只允许漫射阳光进入室内, 避免了直射阳光对 室内环境的影响，提高了室内的热舒适性。

同时，双层构造的组合幕墙墙面之间为 $600 \mathrm{~mm}$ 的空气间层，大大增强建筑表皮的热惰性，保证了室温 的稳定, 这在冬季寒冷的太原地区非常有利于降低车站运行的能耗 [8]。

整个建筑外墙的平均传热系数仅为 $0.39 \mathrm{~W} /(\mathrm{m} 2 \cdot \mathrm{K})$, 远低于现行节能标准的 $0.60 \mathrm{~W} /(\mathrm{m} 2 \cdot \mathrm{K})$ 。外墙主体 采用 $200 \mathrm{~mm}$ 加气混凝土砌块, 主体墙传热系数 $\mathrm{K}=\mathrm{I} .08 \mathrm{~W} /(\mathrm{m} 2 \cdot \mathrm{K})$, 外墙平均传热系数 $\mathrm{K}=\mathrm{I} .26 \mathrm{~W} /(\mathrm{m} 2 \cdot \mathrm{K})$ 。 项目的玻璃幕墙采用双层中空Low $-\mathrm{E}$ 玻璃, 其传热系数 $\mathrm{K}=2.0 \mathrm{~W} /(\mathrm{m} 2 \cdot \mathrm{K})$. 玻璃遮阳系数 $\mathrm{SC}=0$. 48 , 可见 光透射 I: LVt $=0.67$, 玻璃幕墙的气密性等级为 3 级。石材幕墙采用铝合金挂件系统。龙骨采用钢龙骨, 钢材表面采用热镀锌处理, 面板采用 $25 \mathrm{~mm}$ 厚济南青花岗岩, 密封采用石材专用密封胶。立面采用铝合金可 调遮阳板系统。屋顶主体为 $150 \mathrm{~mm}$ 厚钢筋混凝土，保温材料采用 $35 \mathrm{~mm}$ 厚的挤塑聚苯乙烯泡沫塑料板，屋面 
为绿化屋面。屋顶传热系数 $\mathrm{K}=0.67 \mathrm{~W} /(\mathrm{m} 2 \cdot \mathrm{K}))$ 。

\section{3 .3 幕墙系统}

建筑幕墙系统主要由低辐射玻璃、可控玻璃窗和双层仿清砖石材面板构成。正立面东西向采用低辐射 玻璃及双层仿清石材面板可有效减少太阳辐射, 降低东、西晒对室内环境的影响, 从而减少夏季空调负荷。 可控玻璃窗为手动控制和自动控制, 能够确保夏季可开启, 冬季可关闭, 形成夏季通风, 冬季蓄热的室内 环境。

\section{4 建筑自遮阳设计}

由于交通运输线路原因，太原南站主站房横跨东西方向，因此主要入口从东西向进入。站房空间内部 在南北向由于铁路站台雨棚的存在, 给站房的南北向遮阳提供了一定帮助。东西广场作为建筑的主要入口, 由于东西晒的影响, 对建筑内热环境不利。结合建筑造型和设计单元布局, 在东西向分别增加增加一排设 计单元，从设计上不仅创造了过渡的“灰空间”，同时在东西向形成了自然的自遮阳设施。

项目设计时通过生态设计软件ECOTECT模拟分析了站房东西主立面屋檐对夏至日和冬至日各时间点建 筑立面的太阳辐射和遮阳区域影响 [9]。从分析可知, 在夏季至日 $16: 00$ 以后, 太阳光线才照射到西立面; 而在冬至日由于太阳高度角的变化, 对建筑立面的采光和辐射并未产生不利影响。合理的的屋檐出挑长度, 既保证了站房主立面夏季高温时段的自我遮阳效果, 又保证了冬季阳光能够直射到建筑室内, 从而有效的 化解了站房主立面偏西向的不利条件。

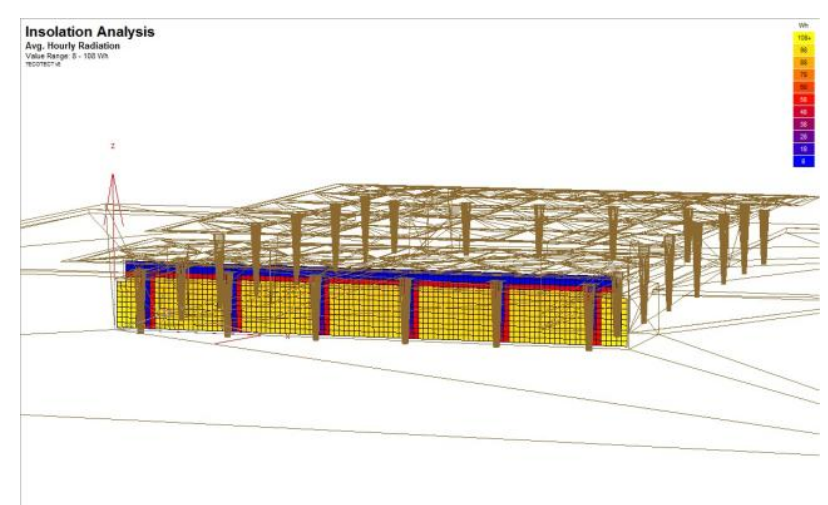

图 3 冬至日 15:00-16:00 西立面太阳辐射示意图

\section{5 地板热辐射采暖}

地板辐射采暖是以温度不高于 $60^{\circ} \mathrm{C}$ 的热水, 在埋置于地板下的盘管系统内循环流动, 加热整个地板, 通过地面均匀地向室内辐射散热的一种供暖方式, 相比传统采暖有无可比拟的优势, 具有舒适、节能、环 保等优点。太原南站大面积采用地板热辐射采暖, 是建立在专业机构针对该技术所做的模拟计算及详细研 究基础之上的 [10]。研究成果显示：以《公共建筑节能设计标准》（GB50189-2005）为对比基准，本项目 的地源热百每年可节省 117.71 标准煤, 年减排C0, 约 $195.5 \mathrm{t}$, 年运行费用可节省 22.3 万元, 动态回收期 11.98 年。由此可见，采用该系统在经济上是合理的，符合目前国家的节能减排政策。

\section{3 结语}

本文基于太原市的当地环境特征，从自然通风、采光，自遮阳系统等方面探寻了太原南站作为典型大 空间建筑在节能方面的可行方向, 同时利用 CFD 模拟技术, 对建筑进行了风环境、太阳辐射模拟, 辅以得 
出最佳设计策略。最后结论发现, 对大空间建筑的通风环境影响因素有: 进排风口风帽的高差、面积, 朝 向; 采光方面的影响有：建筑朝向、天窗采光、遮阳体系的设置; 围护结构节能方面的影响有: 材料的选 择、双层墙体空气层的设置。太原南站作为大空间绿色建筑的一次积极尝试, 从自然通风、自然采光、绿 色照明等自然能源利用方向采取恰当的设计策略, 选取适宜的围护体系, 营造了舒适宜人的室内热环境空 间; 也由于本项目对节能技术的运用，使建筑使用成本大大降低，通过减排二氧化碳保护环境，减轻了能 源供应结构负担, 降低了对能源利用的依赖性。太原南站的设计策略是对大空间建筑设计的一次有益尝试, 可为同类建筑在节能设计上提供思路和方向, 从而实现真正的绿色建筑。

\section{4 致谢}

湖北省教育厅科学技术研究项目（项目编号：Q20151107）

\section{Acknowledgement}

Project of science and technology research by Hubei education department (project number: Q20151107)

\section{参考文献:}

[1] 刘加平等. 绿色建筑概论 [M]. 北京: 中国建筑工业出版社, 2010

[2] 黄涛, 王建廷, 黄城志. “被动优先” 策略在绿色建筑节能设计中的应用难点与对策研究 [J]. 建筑科学, 2016, $32(6), 18-20$

[3] 国家标准《绿色建筑评价标准》(GB/T 50378-2006)

[4] 郭建文，绿色建筑节能设计思考 $[\mathrm{J}]$ ．城市建筑，2014，36, 30-32

［5］山西省公共建筑节能设计标准 DBJ04-241-2006

[6] 黄海静，陈纲。建筑采光节能设计的整体思维 [J]．重庆建筑大学学报，2007，29(4)，19-21

[7] 任海滨. 刍议现代建筑的绿色节能设计 [J]. 山西建筑，2013，39(02)，10-12

[8] Peter, Smith. Architecture in climate of change A Guide to Sustainable Design[M]. Burlington: Architecture Press, 2005.

[9] Edward Sowell F , Philip Haves . Efficient solution strategies for building energy system simulation[J]. Energy and Buildings, 2001, 33, 22-24

[10] Soteris Kalogirou A. Solar thermal collectors and applications [J]. Progress in Energy and Combustion Science, 2004, 30, 32-34

\section{References}

[1] Liu Jia-ping: Introduction to green building [M]. (Beijing: China Building Industry Press, China 2010), p.30-34

[2] Huang Tao, Wang Jian-ting, Huang Zhi-cheng: "Apply difficulties and countermeasure research on green building energy efficiency design in 'Passive priority' strategy" [J]. Building Science, Vol.32 (2016), No.6, p.18-20(in Chinese)

[3] National Standard "Evaluation Standard of Green Building "(GB/T 50378-2006) (in Chinese)

[4] Guo Jian-wen: "Thoughts of green building energy saving design" [J]. Urban Construction, Vol.36 (2014), p.30-32(in Chinese)

[5] Public Building Energy Efficiency Design Standards in Shanxi Province DBJ04-241-2006(in Chinese). 
[6] Huang Hai-jing, Chen Gang. Overall thinking of building lighting energy saving design [J]. Journal of Chongqing University of Architecture, Vol.29 (2007), No.4, p.19-21(in Chinese)

[7] Ren Hai-bin: "Discuss about the green energy-saving design of modern architecture"[J]. Shanxi Architecture, Vol. 39(2013), No.2, p.10-12(in Chinese)

[8] Peter, Smith. Architecture in climate of change A Guide to Sustainable Design [M]. Burlington: Architecture Press,2005.

[9] Edward Sowell F, Philip Haves. Efficient solution strategies for building energy system simulation [J]. Energy and Buildings,2001, 33, 22-24

[10] Soteris Kalogirou A. Solar thermal collectors and applications [J]. Progress in Energy and Combustion Science, 2004, 30, 32-34 